\title{
Fourier Plane Image Combination by Feathering
}

\author{
W. D. Cotton
}

\begin{abstract}
Astronomical objects frequently exhibit structure over a wide range of scales whereas many telescopes, especially interferometer arrays, only sample a limited range of spatial scales. In order to properly image these objects, images from a set of instruments covering the range of scales may be needed. These images then must be combined in a manner to recover all spatial scales. This paper describes the feathering technique for image combination in the Fourier transform plane. Implementations in several packages are discussed and example combinations of single dish and interferometric observations of both simulated and celestial radio emission are given.
\end{abstract}

\section{INTRODUCTION}

$\mathbf{C}$ ELESTIAL images may contain structure on a wide variety of size scales whereas a given radio astronomical instrument may be sensitive to only a limited range of size scales. A common solution to this problem is to image the desired object with a number of different instruments, or configurations of a given instrument, to recover the structure on the total range of size scales needed. These range from single dishes for the largest scales to short baseline interferometers to longer baseline interferometers. Furthermore, each instrument and/or array configuration may have artifacts which are best dealt with using that data-set alone. This paper considers the combination of images derived from several instruments or configurations by the "feathering" technique. The term "feathering" is likely derived from the similarity with bird's feathers which are dense at the center and very light at the edge. In this technique, images are combined in the Fourier transform ("uv") domain by a weighted average of the transform of the various input images in order to extract the most appropriate spatial frequencies from each.

\section{Resolution and Spatial Dynamic Range}

Diffraction limited astronomical instruments have a resolution that is proportional to the diameter of the aperture measured in wavelengths of the light being observed. The range of larger spatial scales to which the instrument is sensitive depends on the details of the instrument and in particular, the distribution and fraction of its aperture which is filled. Filled aperture instruments (AKA "single dishes") sample all spatial frequencies up to those defined by the total aperture. Cost and other practical constraints limit the maximum size, hence resolution of single dishes. On the other hand, interferometers generally sample only a fraction of the spatial frequencies less than that defined by the maximum baseline. The largest scale size that can be imaged is defined by the shortest spacing that is adequately sampled. Interferometers can be constructed to an arbitrary size, hence, resolution but

National Radio Astronomy Observatory, 520 Edgemont Rd., Charlottesville, VA, 22903 USA email: bcotton@nrao.edu practical constraints limit the fraction of the aperture that can be filled thus the largest scale size. Some interferometers such as the VLA and ALMA have a "zoom" capability in which the antennas can be reconfigured to produce a range of resolutions and surface brightness sensitivities. In addition, ALMA has an array (ACA) of smaller antennas arranged in a more compact configuration to measure even lower spatial frequencies. For objects with a wide range of spatial scales, multiple instruments or configurations may be needed.

Every instrument can produce artifacts or spurious features in its images. Techniques to reduce these may be deployed but these are generally specific to a given instrument or configuration. An example of this is a bright source far from the pointing center of an interferometer. Even when not in the field of interest, such a source may produce side-lobes in the field of view that are subject to bandwidth smearing and details of the far antenna pattern. Bandwidth smearing is locally convolutional and the effects of the offending source can largely be removed from the data from a single interferometer or configuration by deconvolution including that source. Artifacts from asymmetries in the antenna pattern are not convolutional but may be greatly reduced by corrections based on known antenna patterns, by "peeling" or "differential gains" [1].

Strong, extended sources whose structure is not adequately sampled by an interferometer will have an extended negative "bowl" surrounding them in derived images. This is because the visibility at the center of the uv plane is the total intensity in the field of view. Thus, dirty images made lacking samples near the center of the uv plane (which are implicitly replaced by zeros) will have an average value of zero causing strong positive regions to be surrounded by negative regions. The purpose of deconvolution is to interpolate between measured visibilities including those near the origin. If the uv coverage is inadequate to allow the deconvolution to recover all of the emission in the source, portions of the bowl will remain.

\section{FEATHERING}

An image may be characterized by the spatial frequencies which are well represented in the image; these correspond to the regions in the Fourier transform ("uv" space) of the image which were well measured by the instrument. Interferometers generally cover a range of spatial frequencies which is limited by the longest and shortest baselines. Single dishes, in principle, measure all spatial frequencies up to those corresponding to the instrument diameter Ideally images with overlapping, well-sampled spatial frequencies can be combined to derive an image reproducing all the spatial frequencies in the initial images.

\footnotetext{
${ }^{1}$ However, spatial filtering to remove atmospheric or instrumental variations may filter out some spatial frequencies
} 
Feathering is the technique of combining images in the uv plane to recover the spatial frequencies in the input images. The images to be combined must be adjusted to a common astrometric and photometric scale and, if FFTs are to be used, interpolated onto a common image grid. The combination of $m$ images $I_{i}$ in the Fourier transform (u-v) domain is a weighted average described by the following:

$$
C(u, v)=\sum_{i=1}^{m} W_{i}(u, v) F T\left(I_{i}\right)(u, v)
$$

where $F T$ denotes the Fourier transform, $W_{i}$ is the weighting function for image $I_{i}$. If there is emission near the edge of the images, tapering the images to zero or similar techniques may be needed to reduce the artifacts (ringing) resulting from the Fourier transform. The feathered image is then:

$$
I(x, y)=N F T^{-1}(C)
$$

where $N$ is a normalization factor. The resolution of the resultant image should be that of the highest resolution input image. While this is a generic technique and is widely implemented, the details, i.e. derivation of $W_{i}$ and $N$ and constraints on the images vary from implementation to implementation.

\section{CONSTRAINTS ON IMAGES}

Images to be combined need to be astrometrically and photometrically aligned. All implementation described below require the images to be astrometrically aligned but differ in whether the photometric alignment is including in the feathering. In a range of cases it is possibly to align the flux density scales by comparing the Fourier transform values in the annulus of overlapping spatial frequencies.

Image combination will work best when the images combined have well sampled overlapping regions of the uvplane. In the case of non-overlap, structure represented by the portions of uv space not sampled will not be well represented in the feathered image. Clearly, combining single dish and VLBI images will not generally be productive.

Using the amplitude ratios in annuli may not always be the best method for adusting the flux density scales but it is well defined for single interferometric pointings and is widely used. An alternative is to use isolated, spatially small features as an adjustment of the astrometric grids is generally also needed and such features can be used for both.

If the interferometer sampling in the uv overlap is really dense then adjustment using the annuli should be relatively robust; unfortunately this is often not the case and the interferometric measurements are less well sampled. Since the comparison is made using the interferometric image rather than the visibility data, the resolution of extended emission will generally bias the amplitudes in the interferometric annulus low. A correction for this will incorrectly increase the relative power in the higher spatial frequencies making smaller scale structures erroneously brighter. The annular method of adjusting flux density scale is more problematic in the case of large scale images needing interferometric mosaics.

The technique described above assumes that each input image has been appropriately filtered to remove power at spatial frequencies beyond the range sampled by the instrument used to derive the image. For interferometric images this constraint is generally that the emission CLEANed is restored with a CLEAN beam that accurately represents the psf of the instrument. Images derived from single dish measurements may not have had similar filtering and may need to have spatial frequencies outside of the telescope aperture filtered before combination.

Interferometric images of sources with extended emission generally need extra care as emission poorly sampled in the uv-plane can lead to image artifacts. Multi-resolution CLEAN can help with reconstructing large-scale emission. In extreme cases, setting a short baseline limit during the imaging can help suppress large waves from very poorly sampled structure. Negative bowls around extended emission are common but should be removed by feathering if the relevant spatial frequencies are obtained from other images.

\section{A. Primary beam correction}

Interferometric images should be "Primary beam corrected" before feathering such that features in the image are at the strength they would have without off-center attenuation of the power pattern of the interferometer elements

\section{B. Mosaics}

Mosaics are suitable candidates for feathering as long as the combination into the mosaic removes the primary beam pattern.

\section{Weighting}

Not all images are created equal and it may be desirable to allow different images to have different weights where their uv regions overlap. A simple relative weight is the inverse variance of the noise. This additional weight is multiplied times the feathering weight.

\section{Spectral cubes}

Feathering spectral line cubes is a straightforward combination of each set of channel images. Cubes must either have a common channelization or be interpolated onto one.

\section{Alternatives to Feathering}

There are several alternatives to combining data from different instruments. Their properties differ and the best technique depends on the data involved. For a broad range of cases, feathering produces acceptable results. See [2] for a discussion on a variety of techniques

\section{A. Combine visibilities}

If several interferometers are to be combined, the visibilities can be combined into a single data-set which is then imaged. Single disk images can be Fourier transformed to produce "pseudo" visibilities. However, the single dish psf (antenna pattern) must first be deconvolved and the result reconvolved with the interferometer antenna pattern. Since the aperture is 
filled, this can be done using a linear deconvolution. AIPS task IM2UV can convert an image into pseudo visibilities.

Special care must be taken when combining data from interferometer arrays with different antenna sizes as the field of view differs. The imaging software must properly correct for the different antenna sizes if a wide field of view or mosaic is to the imaged.

Visibility data is generally "weighted", that is each visibility is given a weight with which it is to be used in the image construction. These weights may need to be adjusted in the visibility combination process to assure that the data makes the appropriate contribution to the derived image.

\section{B. Combine dirty images and deconvolve}

The formation of dirty images is linear so an interferometer image can be linearly combined with other interferometer dirty images or a single dish image. The combined image can then be deconvolved; Maximum Entropy (MEM) is frequently used for this. Miriad task MOSMEM applies this technique.

\section{Use single dish image as model in deconvolution}

A discussion of using the single dish image as an initial model using Maximum Entropy deconvolution is described in [3]

\section{IMPLEMENTATIONS}

\section{A. AIPS Implementation}

The AIPS [4] implementation is in task IMERG and will combine two images. These must be on a common grid and size but IMERG will adjust the flux density scale using the values in a specified annulus of the uv plane. Interior to the specified uv annulus, the lower resolution image is given weight 1 and the higher resolution image a weight 0 . These are reversed outside the annulus and within the annulus an exponential is used to give increasing weight to the higher resolution image. Since the sum of the weights are always 1 , the normalization factor is 1 .

\section{B. CASA Implementation}

The CASA [5] implementation is in task feather and allows combining two images and consists of the following steps:

- Interpolate the low resolution image onto the grid of the high resolution image.

- Fourier transform both images to the uv-plane.

- The weight of the low resolution image is the ratio of the beam area of the high resolution image to that of the low resolution image

- The weight of the high resolution image is $1-w$, where w is the Fourier transform of low resolution CLEAN beam.

- Back transform combined grid to image plane.

- No normalization is documented.

\section{Miriad Implementation}

The Miriad [6] implementation is in task immerge with option 'feather' which allows combining two images with Gaussian psfs. The input images must be on a common grid and size and flux density scale although the program will optionally adjust the lower resolution flux density scale using overlapping spatial frequencies. The weight of the low resolution image is 1 everywhere and that of the high resolution image is the Fourier transform of the low resolution CLEAN beam. The resultant resolution is that of the higher resolution image.

\section{Obit Implementation}

The feathering implementation in Obit[7] follows that described in [8] and is implemented in task Feather which will combine up to 10 images. Feather requires only that the astrometric parameters in each image give the same positions for given features and need not be on the same grid, projection or even equinox.

The photometric (flux density) scale must be the same for all input images. If this cannot be achieved from the calibration of the data, an adjustment can be derived prior to feathering from the average ratio of amplitudes in the overlapping region of uv space.

Images are presumed to have an annular region in the uv-plane which is well sampled and a weighting mask is constructed for each which tapers to zero at lower spatial frequencies. In the case where there is not an overlap in the well sampled regions of uv space, structures corresponding to spatial frequencies not well sampled in any of the input images may be poorly represented in the feathered image, or missing entirely. The weighting mask of the lowest resolution image is not tapered to its center. Feather depends on the "CLEAN" beam size given in each image descriptor accurately reflecting the resolution of the image. The various steps in feathering are described in the following.

\section{1) Re-sample images}

The first step is to re-sample images with resolutions less than the maximum to the grid of the maximum resolution image with sufficient zero padding on the outside to allow an efficient FFT. The interpolation uses the Lagrangian technique in $2 \mathrm{D}$ to interpolate the pixels in the lower resolution images at the locations of the highest resolution image using a $5 \times 5$ pixel kernel.

\section{2) Generate weighting masks}

For each resolution except the lowest, a real weighting mask is generated with a Gaussian hole in the center representing the spatial frequency range of the next lowest resolution. A sampling mask representing the spatial frequencies of each image is generated by:

a) Create image with the CLEAN beam at the center

b) FFT

c) Take real part

d) normalize to 1 at $(0,0)$ spatial frequency

\footnotetext{
${ }^{2}$ http://www.cv.nrao.edu/ bcotton/Obit.html
} 
The weighting mask for each image $i$ is

$$
w_{e i g h t \_m a s k_{i}}=1.0-\text { sampling_mask }_{i+1}
$$

where $i+1$ indicates the next lowest resolution. The weighting mask for the lowest resolution is $1.0 \mathrm{ev-}$ erywhere. The weighting masks are multiplied by the weights assigned to the input images.

3) FFT

Each image is FFTed to the uv plane

4) Weight

Multiply Fourier transform of image by its weighting mask.

5) Accumulate

Sum the Fourier transform of the images times weight.

6) Inverse FFT

Fourier transform back to the image plane.

7) Normalize

The normalization factor is determined by repeating the process but replacing the image with its corresponding CLEAN beam. The normalization factor is $1.0 /$ fluxdensity(center pixel) of the feathered beam.

\section{EXAMPLES}

\section{A. Simulation}

To illustrate the power of this technique, noiseless synthetic data sets were derived for the model source distribution shown in Figure 1. A simulated single dish image was derived by convolving the model image with the resolution of the single dish. To simulate an interferometric image, a sample full track, VLA-like array uv coverage was generated (Obit/UVSim) and the Fourier transform of the model was evaluated at the locations in the data-set. These data were then imaged with multi-resolution CLEAN (Obit/Imager). The simulated single dish and interferometric images are shown in Figure 2. The very extended emission is only visible in the single dish representation and the most compact emission only visible in the interferometric version. These two images were then feathered together with equal weights using Obit/Feather giving the image shown in Figure 3. The interferometer image contains $7 \%$ of the initial model flux density as derived by an integral over the image whereas the single dish image has $96 \%$. The combined, feathered image also contains $96 \%$ of the initial model flux density. The missing $4 \%$ is due to truncating the single dish image.

\section{B. Celestial Example}

For a celestial example, single dish observations of the Galactic center at $20 \mathrm{~cm}$ wavelength made with the GBT [8] are combined with a VLA mosaic at the same wavelength [9], [10]. Figure 4 shows a subset of the image around the Galactic center with the single dish and interferometric images on top and the combined image on the bottom. The images were aligned astrometrically and photometrically using several strong, isolated HII regions. The missing short spacing in the VLA image leave a deep bowl around the image which is filled in by adding the GBT image. Due to the strong, wide spread emission in this part of the sky, integrated flux densities

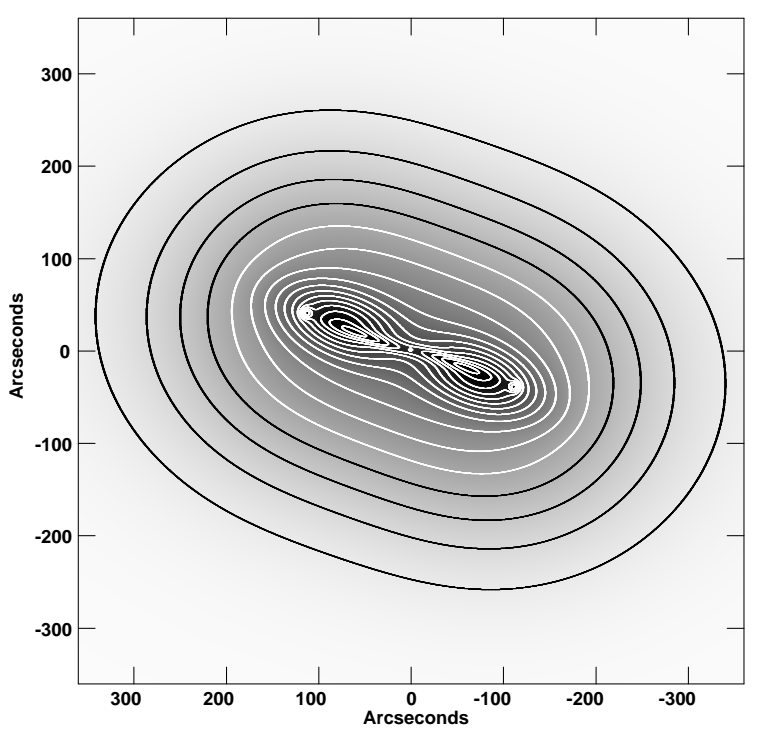

Fig. 1. Model source shown in reversed grayscale with linearly spaced contours overlaid.

cannot be accurately determined from this data. Note: the VLA image is a mosaic derived in AIPS using a suboptimal image plane "feathering" to combine the images resulting in the lines visible between adjacent pointings. These artifacts are greatly reduced if the spatially overlapping images are feathered in the sense of weighting that decreases to zero at the edge.

\section{DISCUSSION}

The feathering technique for image combination in the Fourier plane is described and examples of its use shown. In an example using simulated data, the feathered image recovered all of the flux density in the low resolution image. In the celestial example a deep negative bowl around a bright region of emission in the interferometric image is removed by adding the single dish data.

The feathering technique as implemented in the Obit package described here allows an arbitrary number of images at different scales to be combined. This allows the best approach to reducing artifacts to be used for each of the multiple interferometric and single dish images before their combination.

\section{ACKNOWLEDGMENT}

The author would like the thank the anonymous referee for comments leading to an improved paper. The National Radio Astronomy Observatory (NRAO) is operated by Associated 

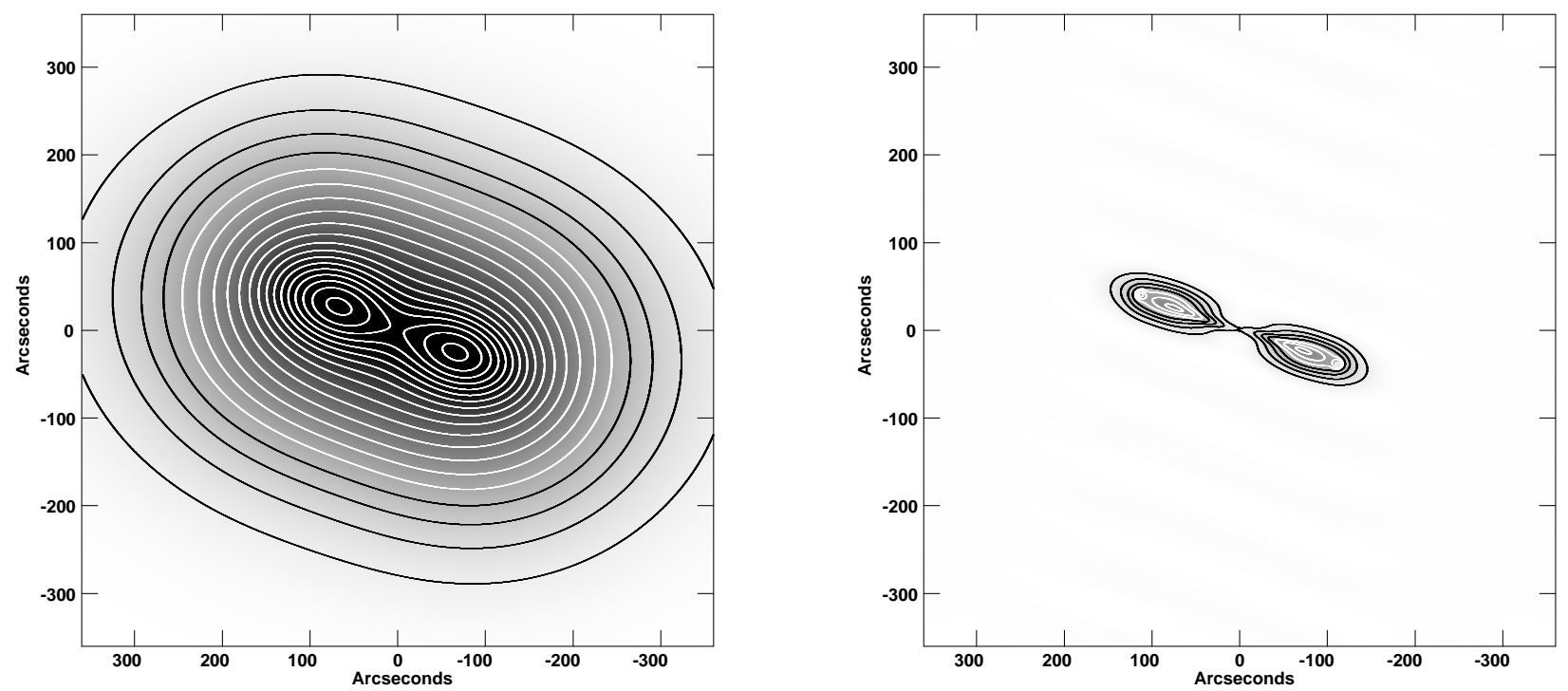

Fig. 2. On left is the model source as observed with a single dish and on the right with an interferometer. Contours are at the same levels as Figure 1

Universities Inc., under cooperative agreement with the National Science Foundation.

\section{REFERENCES}

[1] O. M. Smirnov, "Revisiting the radio interferometer measurement equation. III. Addressing direction-dependent effects in $21 \mathrm{~cm}$ WSRT observations of 3C 147," Astron.\&Astrophy., vol. 527, p. A108, Mar. 2011.

[2] S. Stanimirovic, "Short-Spacings Correction from the Single-Dish Perspective," in Single-Dish Radio Astronomy: Techniques and Applications, ser. Astronomical Society of the Pacific Conference Series, S. Stanimirovic, D. Altschuler, P. Goldsmith, and C. Salter, Eds., vol. 278, Dec. 2002, pp. 375-396.

[3] T. J. Cornwell, "Radio-interferometric imaging of very large objects," Astron.\&Astrophy., vol. 202, pp. 316-321, Aug. 1988.

[4] E. W. Greisen, "The Astronomical Image Processing System." in Acquisition, Processing and Archiving of Astronomical Images, G. Longo and G. Sedmak, Eds., 1990, pp. 125-142.

[5] J. P. McMullin, B. Waters, D. Schiebel, W. Young, and K. Golap, "CASA Architecture and Applications," in Astronomical Data Analysis Software and Systems XVI, ser. Astronomical Society of the Pacific Conference Series, S. R. A., F. Hill, and D. J. Bell, Eds., vol. 376, 2007, pp. 127-+.

[6] S. R. J., P. J. Teuben, and M. C. H. Wright, "A retrospective view of Miriad," in Astronomical Data Analysis Software and Systems IV, ser. Astronomical Society of the Pacific Conference Series, R. Shaw, H. E. Payne, and J. J. E. Hayes, Eds., vol. 77, 1995, pp. 433-+.

[7] W. D. Cotton, "Obit: A Development Environment for Astronomical Algorithms," PASP, vol. 120, p. 439, 2008.

[8] F. Yusef-Zadeh, J. W. Hewitt, and W. Cotton, "A 20 Centimeter Survey of the Galactic Center Region. I. Detection of Numerous Linear Filaments," ApJS, vol. 155, pp. 421-550, Dec. 2004.

[9] C. Law, F. Yusef-Zadeh, and W. D. Cotton, "A Wide-Area VLA Continuum Survey near the Galactic Center at 6 and $20 \mathrm{~cm}$ Wavelengths," ApJS, vol. 177, p. 515, 2008.
[10] C. Law, F. Yusef-Zadeh, W. D. Cotton, and R. Maddalena, "Green Bank Telescope Multiwavelength Survey of the Galactic Center Region," ApJS, vol. 177, p. 255, 2008 


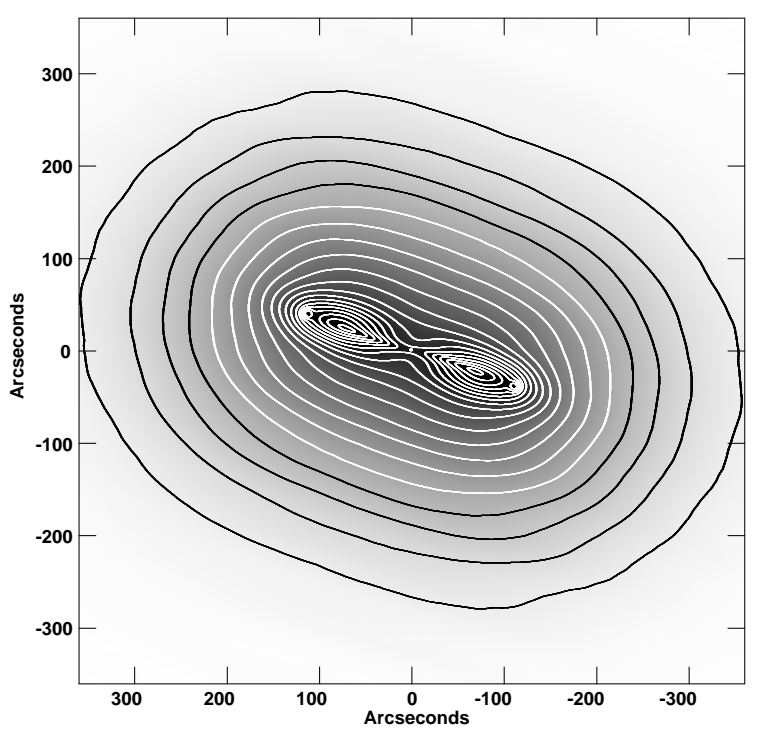

Fig. 3. The feathered combination of the images in Figure 2 Contours are at the same levels as Figure 1 

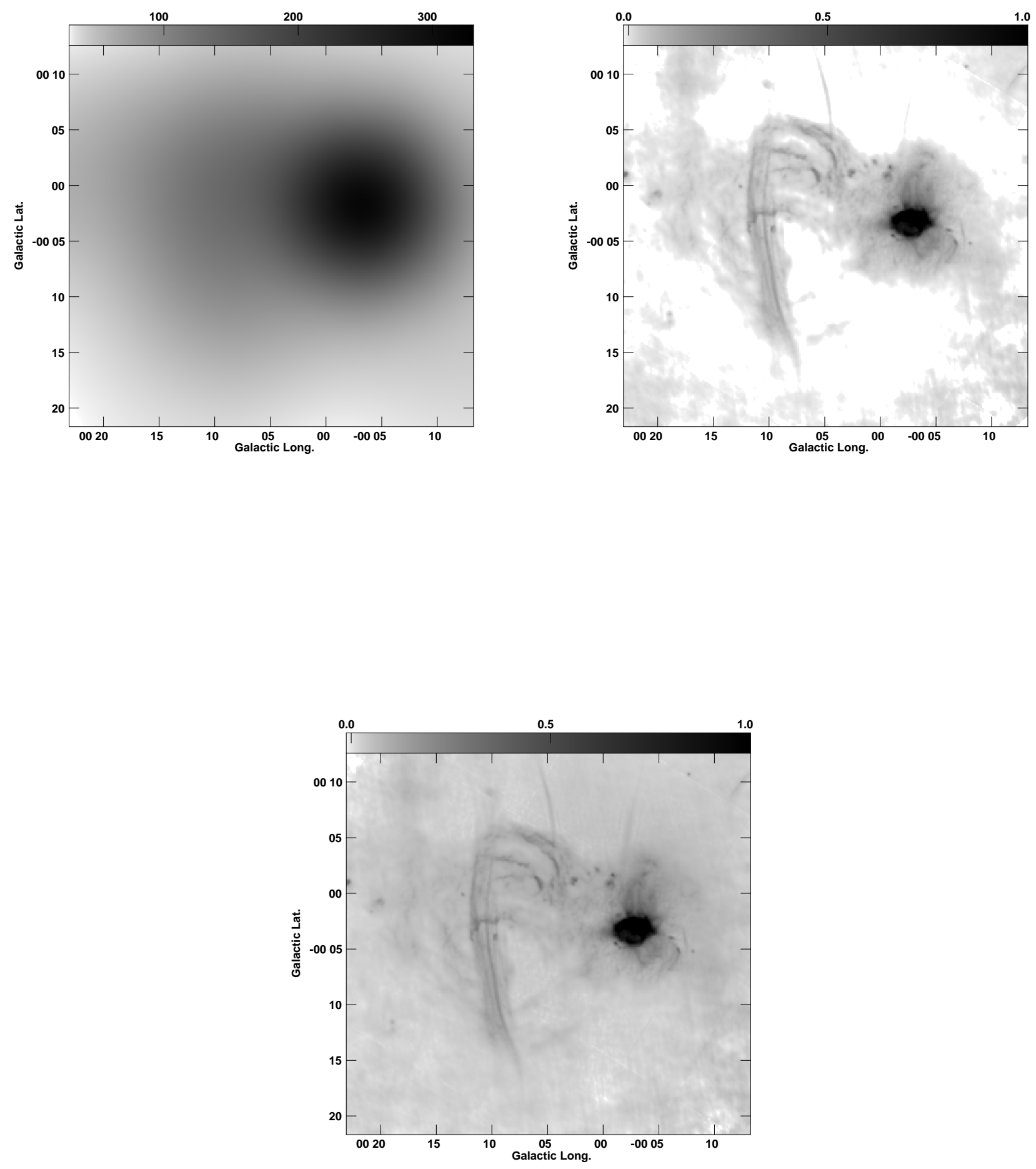

Fig. 4. Top: left is the GBT single dish image and on the right the VLA interferometric image. Grayscale stretch is square root with scale bar at top. Bottom: The feathered combination of the images. Grayscale stretch the same as top right. Images are given as negative grayscale. 